IC/HEP/96-3

29th April, 1996

\title{
Defect evolution in silicon detector material
}

\author{
B. C. MacEvoy* \\ Blackett Laboratory, Imperial College, London SW7 2BZ, UK
}

\begin{abstract}
A numerical kinetics model has been used to investigate the evolution of complex defects in high resistivity silicon detector material during fast neutron irradiation to levels expected at the CERN LHC. The complex $\mathrm{V}_{2} \mathrm{O}$ is identified as a candidate for a deep-level acceptor state which gives rise to experimentally observed changes in the effective doping concentration. The importance of the initial oxygen impurity concentration in determining the radiation tolerance of the detectors is demonstrated. The characteristics of devices heavily irradiated with ${ }^{60} \mathrm{Co}$ photons are modelled satisfactorily by using a semiconductor simulation in conjunction with the kinetics model. It is postulated that inter-defect transitions between divacancy states in the terminal damage clusters are responsible for apparent discrepancies in the modelling of data from neutron-irradiated devices. This mechanism (if correct) may have important consequences for the prospects of "defect-engineering" a radiation hard device.
\end{abstract}

* Tel: +44 1715947797

Fax: +441718238830

E-mail: b.macevoy@ic.ac.uk

Submitted to the International Conference on Radiation Effects on Semiconductor Materials, Detectors and Devices, Florence, Italy, March 6-8 1996 


\section{Introduction}

Silicon detectors will be widely used in experiments at the CERN Large Hadron Collider where high radiation levels will cause significant bulk damage. It is anticipated that microstrip layers will receive hadron fluences equivalent to $\sim 10^{14} \mathrm{~cm}^{-2} 1 \mathrm{MeV}$ neutrons while inner pixel layers will receive even higher fluences $[1,2]$. In addition to increased leakage currents, changes in the effective doping concentration $\left(\mathrm{N}_{\text {eff }}\right)$ have been observed which represent the limiting factor for longterm detector operation [3,4]. It is found that n-type detectors become progressively less n-type with increasing fluence until they invert to effectively p-type at around $10^{13} \mathrm{n} . \mathrm{cm}^{-2}$. Beyond inversion the devices continue to become more p-type under further irradiation, apparently without limit. The depletion voltage required to operate a silicon detector is directly proportional to $\mathrm{N}_{\text {eff, }}$ hence at high fluences $\mathrm{N}_{\text {eff }}$ can be such that the required voltage exceeds the breakdown voltage of the device and efficient operation is no longer possible.

Until recently these changes in doping concentration were poorly understood. It had been hypothesised that the behaviour of $\mathrm{N}_{\mathrm{eff}}$ is due to donor removal (by the attachment of radiation-induced vacancies to phosphorus atoms) and shallow acceptor creation [5]. However, recent Deep Level Transient Spectroscopy (DLTS) measurements have shown that the phosphorus removal rate is some 30 times less than required [6]. Furthermore, no candidate for the shallow acceptor state has ever been identified. A more plausible hypothesis is that the introduction of deep-level acceptor states causes n-type silicon to become effectively p-type under bias [6]. A semiconductor simulation which includes a single acceptor in the Poisson equation has been successfully used to describe the observed evolution of $\mathrm{N}_{\text {eff }}$ with fluence for both n-type and p-type detector material. Figure 1 shows the introduction rate required to describe the $\mathrm{N}_{\text {eff }}$ data correctly as a function of acceptor position relative to mid-gap.

\section{Defect kinetics model}

A more complete understanding can be achieved by numerical calculations of the evolution of defect complexes formed during irradiation. The primary defects produced by bulk damage are vacancies $(\mathrm{V})$, self-interstitials $(\mathrm{I})$ and divacancies $\left(\mathrm{V}_{2}\right)$. The divacancies are static at temperatures up to $\sim 600 \mathrm{~K}$. The vacancies and interstitials are highly mobile at room temperature and those which escape 
recombination diffuse through the crystal until they are trapped at impurity atoms (particularly oxygen and carbon) or other defects.

These trapping events may be thought of in terms of quasi-chemical "reactions". The reaction rates are determined by the concentrations of impurities and defects and their relative capture radii. Davies et al. [7] have explained the optical absorption spectra of electron-irradiated silicon by using a small number of reactions. A kinetics model, based on this work but suitably modified, has been used to investigate defect evolution during fast neutron and gamma irradiation [8]. The reactions are:

\section{I reactions}

PKA cluster

$\mathrm{I}+\mathrm{V} \rightarrow \mathrm{Si}$

Diffusion reactions

$\mathrm{I}+\mathrm{C}_{\mathrm{S}} \rightarrow \mathrm{C}_{\mathrm{i}}$

$\mathrm{I}+\mathrm{CC} \rightarrow \mathrm{CCI}$

$\mathrm{I}+\mathrm{CCI} \rightarrow \mathrm{CCII}$

$\mathrm{I}+\mathrm{CO} \rightarrow \mathrm{COI}$

$\mathrm{I}+\mathrm{COI} \rightarrow \mathrm{COII}$

$\mathrm{I}+\mathrm{V}_{2} \rightarrow \mathrm{V}$

$\mathrm{I}+\mathrm{VP} \rightarrow \mathrm{P}$

\section{$\mathrm{V}$ reactions}

$$
\mathrm{V}+\mathrm{V} \rightarrow \mathrm{V}_{2}
$$

$\mathrm{V}+\mathrm{V} \rightarrow \mathrm{V}_{2}$

$\mathrm{C}_{\mathrm{i}}+\mathrm{C}_{\mathrm{s}} \rightarrow \mathrm{CC}$

$\mathrm{V}+\mathrm{V}_{2} \rightarrow \mathrm{V}_{3}$

$\mathrm{C}_{\mathrm{i}}+\mathrm{O} \rightarrow \mathrm{CO}$

$\mathrm{V}+\mathrm{O} \rightarrow \mathrm{VO}$

$\mathrm{V}+\mathrm{VO} \rightarrow \mathrm{V}_{2} \mathrm{O}$

$\mathrm{V}+\mathrm{P} \rightarrow \mathrm{VP}$

\section{$C_{i}$ reactions}

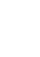

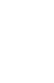




\section{Deep acceptor candidates}

The points superposed upon Figure 1 are the predictions of the kinetics model for the introduction rates of the acceptor complexes. The energy levels have been taken from [10] and [11]. The energy levels of the species CCI, COI etc. are unknown. However, the evolution of $\mathrm{N}_{\text {eff }}$ with fluence is the same at room temperature as at $0^{\circ} \mathrm{C}$ [12] where these complexes cannot be formed. They are not therefore considered important in determining $\mathrm{N}_{\text {eff }}$. The strongest candidate for the deep acceptor state is the divacancy-oxygen $\left(\mathrm{V}_{2} \mathrm{O}\right)$ complex $[10,13]$.

Figure 2 examines the predicted influence of the initial impurity concentrations upon the concentration of $\mathrm{V}_{2} \mathrm{O}$ after a fluence of $10^{14}$ n.cm ${ }^{-2}$. It is seen that a factor of $\sim 10$ increase in the initial oxygen concentration to $\sim 5 \times 10^{16} \mathrm{~cm}^{-3}$ leads to a large reduction in $\mathrm{V}_{2} \mathrm{O}$ concentration. This is because the oxygen getters the vacancies produced during irradiation and suppresses the channel $\mathrm{V}+\mathrm{VO} \rightarrow \mathrm{V}_{2} \mathrm{O}$. Hence the fabrication of devices on oxygenated material should enhance detector radiation tolerance. The growth of oxygenated 3 inch monocrystal float-zone ingots is presently under way in order that direct tests of this important prediction can be made.

\section{Modelling data from gamma-irradiated devices}

In the case of irradiation with ${ }^{60}$ Co photons the struck silicon atom has a very much lower recoil energy than in the case of fast neutrons. The radiation damage therefore consists of a uniform distribution of isolated point defects rather than the dense disordered regions which are caused by the interaction of heavy particles. Results from gamma-irradiated devices are useful in that they provide a virtually independent check of the kinetics model predictions. The introduction rates for ${ }^{60} \mathrm{Co}$ photons required for modelling have been measured as $\eta_{\mathrm{V}}=1.2 \times 10^{-3} \mathrm{~cm}^{-1}$, $\eta_{\mathrm{V} 2}=2.8 \times 10^{-5} \mathrm{~cm}^{-1}[14]$.

The plots in Figure 3 show the measured evolution of $\mathrm{N}_{\text {eff }}$ and dark current $\left(\mathrm{I}_{\mathrm{d}}\right)$ during ${ }^{60} \mathrm{Co}$ photon irradiation. $\mathrm{N}_{\text {eff }}$ was deduced from the capacitance-voltage characteristic using the standard procedure. The results shown are typical of a number of detectors investigated; all devices were observed to undergo type inversion at a dose of $100 \mathrm{MRad}$. The leakage current results yield a damage constant of $(7.0 \pm 1.0) \times 10^{-23}$ A.cm -1 at $20^{\circ} \mathrm{C}$ (no correction for self-annealing). 
Values for both $\mathrm{N}_{\text {eff }}$ and $\mathrm{I}_{\mathrm{d}}$ may be derived from the defect concentrations predicted by the kinetics model by solving the Poisson and continuity equations in conjunction with Shockley-Read-Hall (SRH) semiconductor statistics. The unknown quantities which enter the calculations are the ratios of the capture cross-sections for electrons and holes $\left(\sigma_{e} / \sigma_{h}\right)$ for each defect (important in the determination of $\mathrm{N}_{\text {eff }}$ ) and absolute values for the cross-sections (necessary for the calculation of $I_{d}$ ). Throughout it is assumed that $\sigma_{\mathrm{e}} / \sigma_{\mathrm{h}} \sim 1$ for all defect species.

The prediction for $\mathrm{N}_{\text {eff }}$ is superposed on Figure 3a. The complex $\mathrm{V}_{2} \mathrm{O}$ is found to

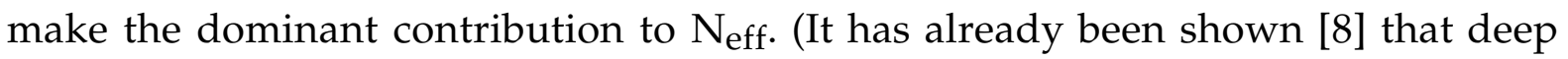
acceptors are present to a significant extent in gamma-irradiated material). The prediction for dark current is shown in Figure 3b. Due to the proximity of the defect to mid-gap the concentration of $\mathrm{V}_{2} \mathrm{O}$ determines the magnitude of $\mathrm{I}_{\mathrm{d}}$. The line shown corresponds to $\sigma_{\mathrm{h}}\left(\mathrm{V}_{2} \mathrm{O}\right) \sim 2 \times 10^{-16} \mathrm{~cm}^{2}$. The agreement between the model and the experimental data for the electrical characteristics is remarkably good.

\section{Modelling data from neutron-irradiated devices}

A similar calculation has been used to predict the evolution of doping concentration and leakage current for neutron irradiation (Figure 4). The modelling parameters are fixed by the gamma results. It is seen that the prediction of $\mathrm{N}_{\text {eff }}$ is poor. Moreover, the leakage current is consistently underestimated by around 2 orders of magnitude for fluences between $3 \times 10^{12}$ and $3 \times 10^{14}$ n.cm-2.

Given that the only discernible difference between ${ }^{60}$ Co photon damage and fast neutron damage is the production of defect clusters in the case of the latter, it is likely that the explanation for this apparent failure of the model lies in a consideration of the defects within the clusters. Simple calculations indicate that the local divacancy density within a typical cluster is $\sim 10^{18} \mathrm{~cm}^{-3}$. Since the extent of bonding distortion caused by $\mathrm{V}_{2}$ can be as large as $\sim 10 \AA$ direct carrier transitions between some of the divacancies are possible. There is some evidence in the literature for such inter-defect transitions [15,16].

The SRH calculation assumes that the only communication between defect centres is the indirect statistical link via the occupation of the bands. It is clear that this approach is not valid in the clusters. The divacancy has 3 states in the forbidden gap: a donor $(+/ 0)$ at $\mathrm{E}_{\mathrm{V}}+0.20 \mathrm{eV}$; a first acceptor $(0 /-)$ at $\mathrm{E}_{\mathrm{C}}-0.41 \mathrm{eV}$; and a second acceptor $(-/=)$ at $E_{C}-0.23 \mathrm{eV}$. By writing general expressions for the rates of emission 
and capture between any two of these states it is possible to solve for the occupancy of each state as a function of local divacancy density in the cluster. (The full calculation is described in [17]).

The occupancy of the first acceptor (denoted $f_{2}$ ) is significantly enhanced over the value predicted by the SRH calculation (Figure 5a). For a divacancy density of $10^{18} \mathrm{~cm}^{-3}$ the contribution of the filled acceptor to $\mathrm{N}_{\text {eff }}$ at a fluence of $5 \times 10^{13} \mathrm{n} . \mathrm{cm}^{-2}$ is $\sim 5 \times 10^{11} \mathrm{~cm}^{-3}$. The results for the leakage current are shown in Figure $5 \mathrm{~b}$. The value of the damage constant $\alpha$ is predicted to be $\sim 10^{-16} \mathrm{~A} . \mathrm{cm}^{-1}$ at $300 \mathrm{~K}$ which is in close agreement with what is actually observed [4]. The inter-defect mechanism can therefore make a significant contribution to both $\mathrm{N}_{\text {eff }}$ and $\mathrm{I}_{\mathrm{d}}$. This may pose a serious obstacle for the "defect-engineering" of a radiation-hard detector because the introduction rate of the divacancy (being a primary defect) is material-independent.

\section{Summary and conclusions}

A numerical model has been used to investigate the evolution of complex defects in silicon detector material during ${ }^{60} \mathrm{Co}$ photon and fast neutron irradiation. The conclusions drawn from this study are as follows:

The divacancy-oxygen $\left(\mathrm{V}_{2} \mathrm{O}\right)$ complex is a candidate for a deep-level acceptor state which makes a significant contribution to the observed doping changes during neutron irradiation. This same complex is almost solely responsible for the changes in $\mathrm{N}_{\text {eff }}$ seen during gamma irradiation;

A high oxygen concentration is predicted to enhance radiation tolerance by suppressing $\mathrm{V}_{2} \mathrm{O}$ production. Direct tests of this hypothesis are vital;

Divacancy coupling in the terminal clusters can make a significant contribution to both $\mathrm{N}_{\text {eff }}$ and the dark current in the case of heavy particle irradiation. If correct, this mechanism has serious consequences for the prospects of engineering a radiation-tolerant device.

Research is currently under way in order to further test the existing predictions of the model and to devise new strategies for the hardening of detector material. 


\section{Acknowledgements}

Valuable discussions with Professor G. Davies, Dr. S. Watts and Dr. J. Matheson are gratefully acknowledged. Thanks, as always, are due to Dr. K. Gill and Dr. G. Hall for their continuing contributions to this work. 


\section{References}

[1] Atlas Technical Proposal, CERN/LHCC 94-43 (December 1994).

[2] CMS Technical Proposal, CERN/LHCC 94-38 (December 1994).

[3] RD2 collaboration. Electrical properties and charge collection efficiency for neutronirradiated $p$-type and n-type silicon detectors. Nucl. Phys. B (Proc. Suppl.) 32 (1993) 415-424.

[4] K. Gill et al. Radiation damage by neutrons and photons to silicon detectors. Nucl. Instr. and Meth. A 322 (1992) 177-188.

[5] E. Fretwurst et al. Nucl. Instr. and Meth. A 326 (1993) 326.

[6] J. Matheson, M. Robbins, S. J. Watts. The effect of radiation induced defects on the performance of high resistivity silicon diodes. CERN RD20/TN/36 (1994) - to be published.

Prior to publication this paper can be obtained from the authors at:

Physics Department, Brunel University, Uxbridge, Middlesex UB8 3PH, UK.

[7] G. Davies et al. A model for radiation damage effects in carbon-doped crystalline silicon. Semicond. Sci. Technol. 2 (1987) 524-532.

[8] B. C. MacEvoy, G. Hall, K. Gill. Defect evolution in irradiated silicon detector material. IC/HEP/95-8 (1995). To be published in Nucl. Instr. and Meth. A

Prior to publication this paper can be obtained from the authors at:

Blackett Laboratory, Imperial College, London SW7 2BZ, UK.

[9] R. A. Woolley et al. Mater. Sci. Forum 10-12 (1986) 929-934.

[10] Y. H. Lee, T. D. Bilash and J. W. Corbett. Radiat. Eff. 29 (1976) 7.

[11] Numerical data and functional relationships in science and technology, Landolt Bornstein, vol. III 22b (1990), Springer-Verlag, Berlin.

[12] F. Lemeilleur et al. Study of characteristics of silicon detectors irradiated with 24 $\mathrm{GeV} / \mathrm{c}$ protons between $-20^{\circ} \mathrm{C}$ and $+20^{\circ} \mathrm{C}$. Nucl. Instr. and Meth. A 360 (1995) 438-444.

[13] Y. H. Lee and J. W. Corbett. EPR studies of defects in electron-irradiated silicon: A triplet state of vacancy-oxygen complexes. Phys. Rev. B 13 (1976) 2653.

[14] Private communication, Dr. J. Matheson, Physics Department, Brunel University, Uxbridge, Middlesex UB8 3PH, UK.

[15] W. M. Chen et al. Direct observation of Intercenter Charge Transfer in Dominant Nonradiative Recombination Channels in Silicon. Phys. Rev. Lett. 67 No. 14 (1991) 1914.

[16] A. Schenk, U. Krumbein. Coupled defect-level recombination: Theory and application to anomalous diode characteristics. J. Appl. Phys. 78 (1995) 3185. 
[17] K. Gill, G. Hall, B. MacEvoy. Divacancy clustering and the origin of leakage current in bulk damaged silicon detectors. CERN CMS/TN/96-17 (1996) - to be published.

Prior to publication this paper can be obtained from:

Dr. K. Gill, PPE Division, CERN, CH-1211 Geneva 23, Switzerland. 


\section{Figure captions}

Figure 1: The lines ascending to the right show the introduction rate required for an acceptor state at a given energy level to describe the $\mathrm{N}_{\text {eff }}$ versus fluence data correctly for different assumptions of electron and hole capture cross-sections [6]. Circles show known acceptor introduction rates from the model.

Figure 2: Contour plot of $\mathrm{V}_{2} \mathrm{O}$ concentration (units - per cubic centimetre) after a fluence of $1 \times 10^{14} \mathrm{n} . \mathrm{cm}^{-2}$ as a function of the initial oxygen and carbon impurity concentrations.

Figure 3: Comparison between model predictions and measured characteristics of a typical device for gamma irradiation: (a) Evolution of $\mathrm{N}_{\text {eff; }}$ (b) Evolution of volumetric dark current.

Figure 4: Comparison between model predictions and measured device characteristics for $1 \mathrm{MeV}$ neutron irradiation: (a) Evolution of $\mathrm{N}_{\text {eff; }}$ (b) Evolution of volumetric dark current.

Figure 5: Consequences of inter-divacancy communication as a function of density in the clusters: (a) Enhancement of first-acceptor occupancy over SRH value; (b) Predicted cluster dark current and damage constant. 


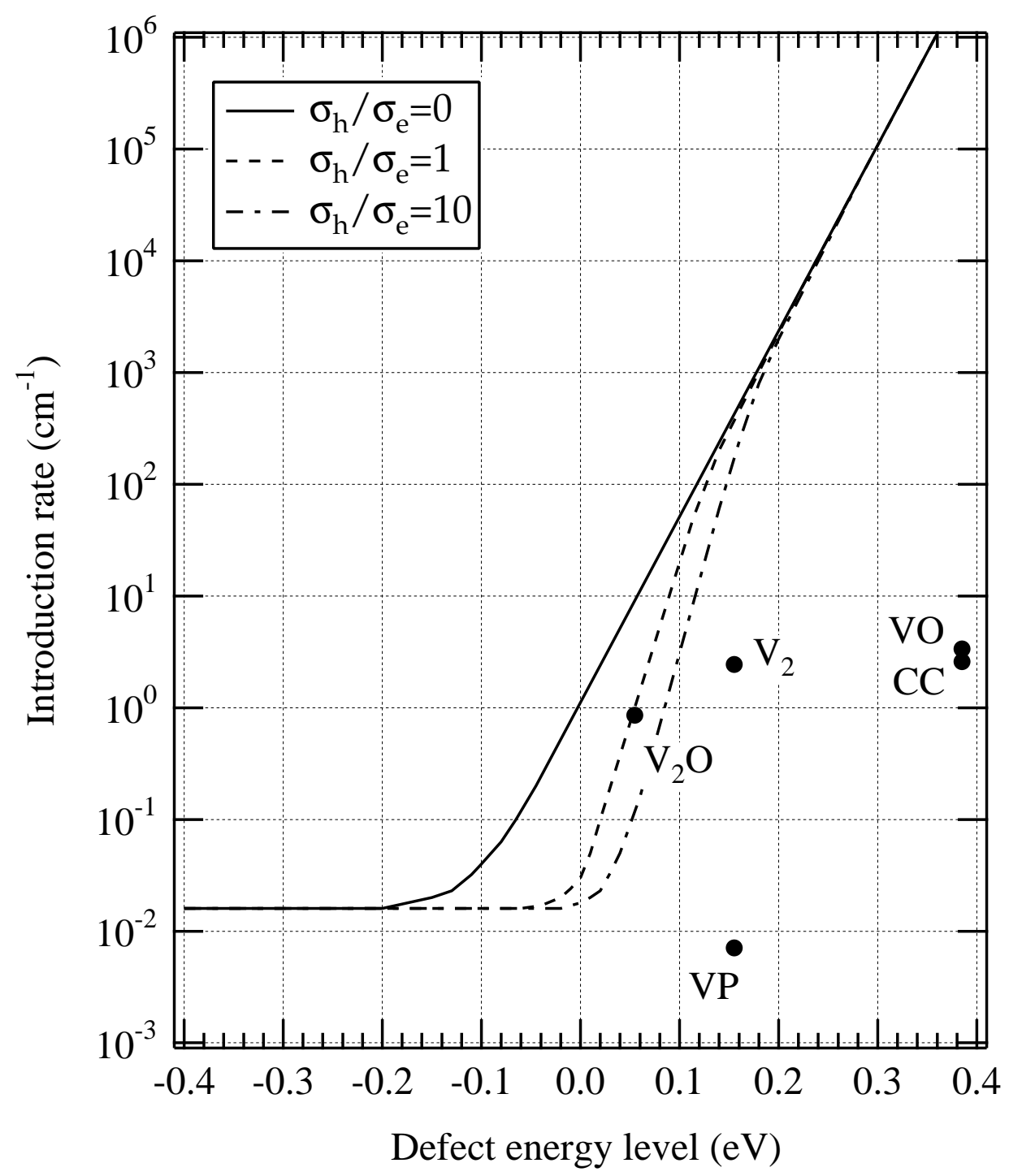

Figure 1 


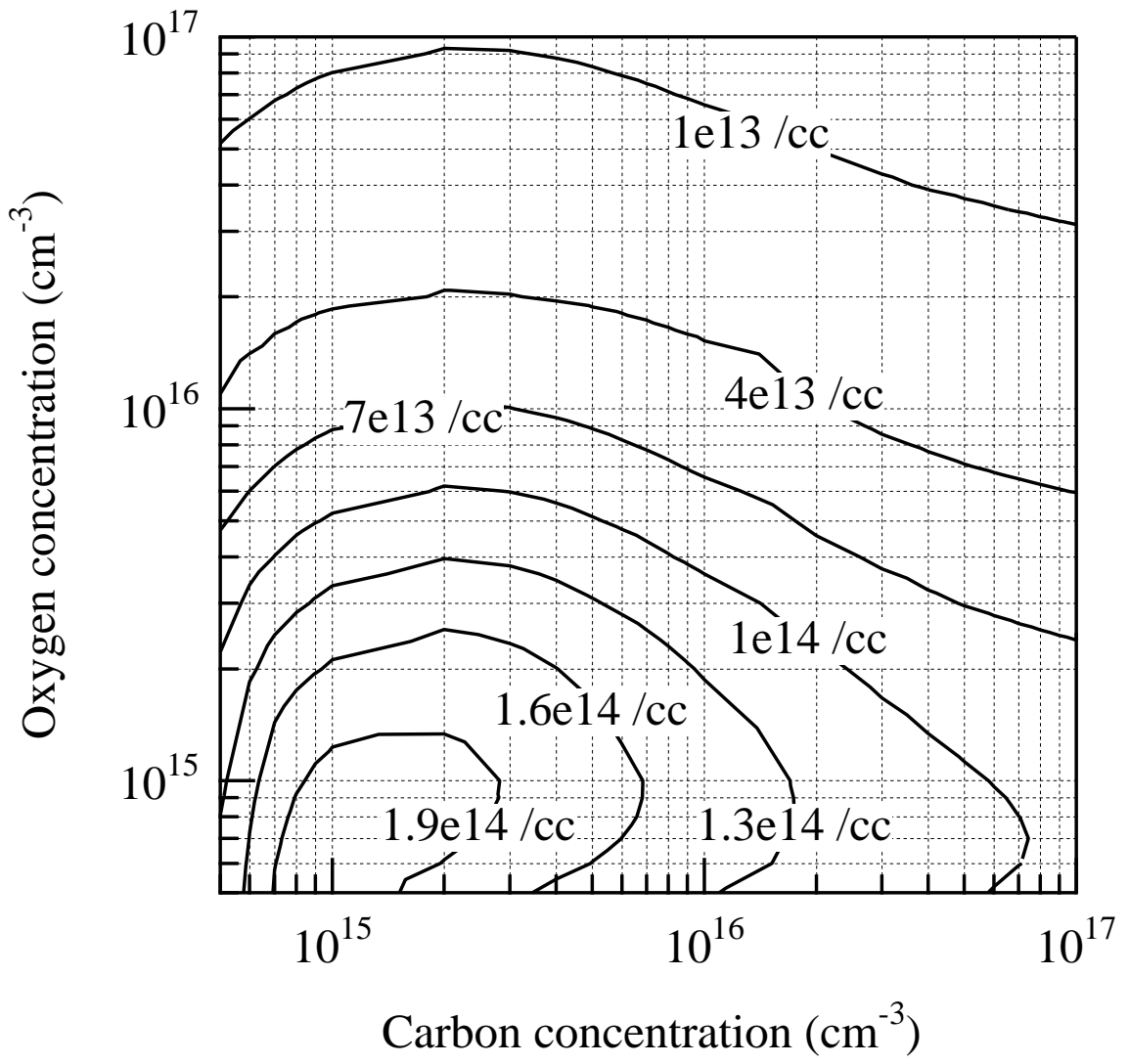

Figure 2 


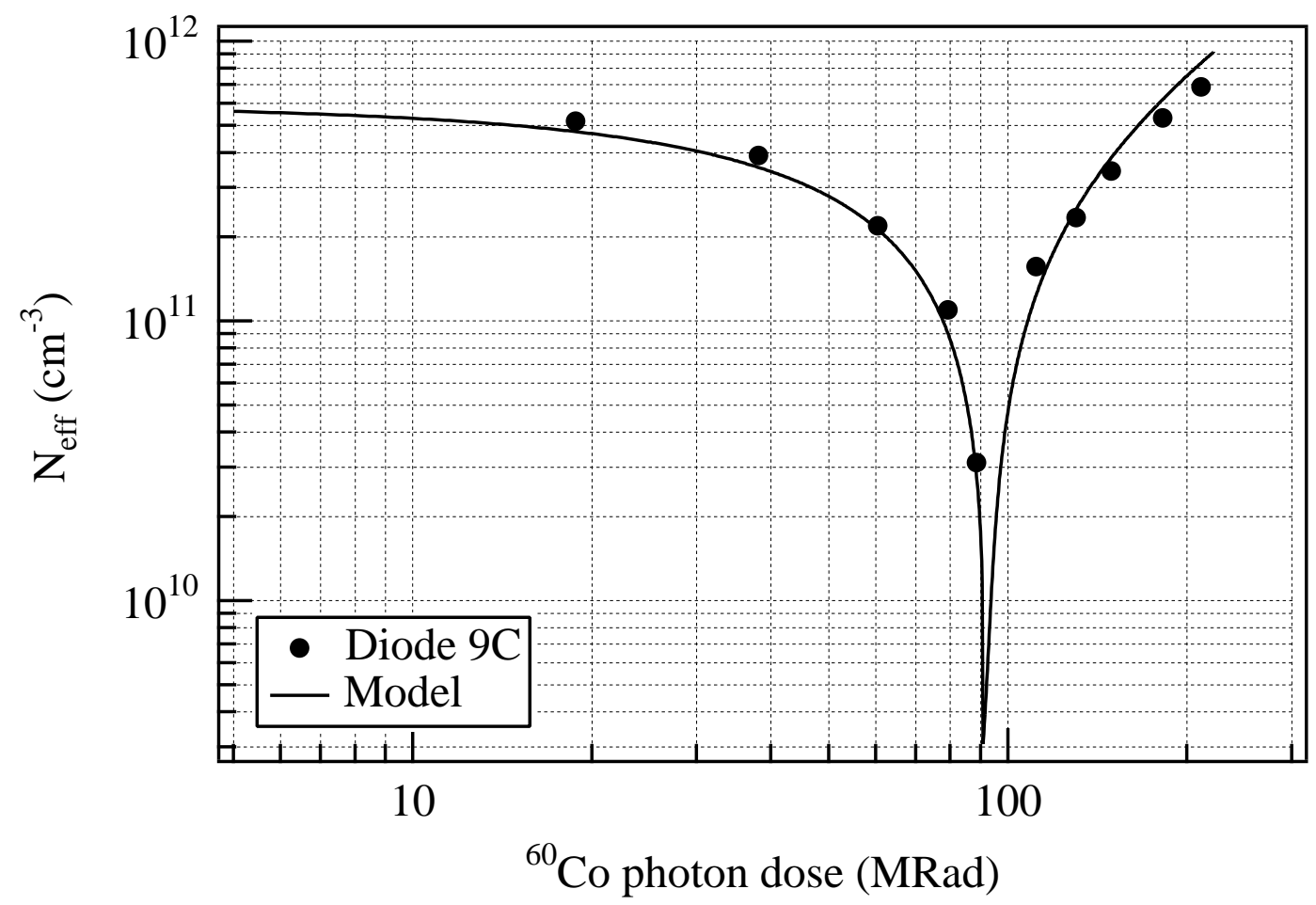

Figure $3 a$ 


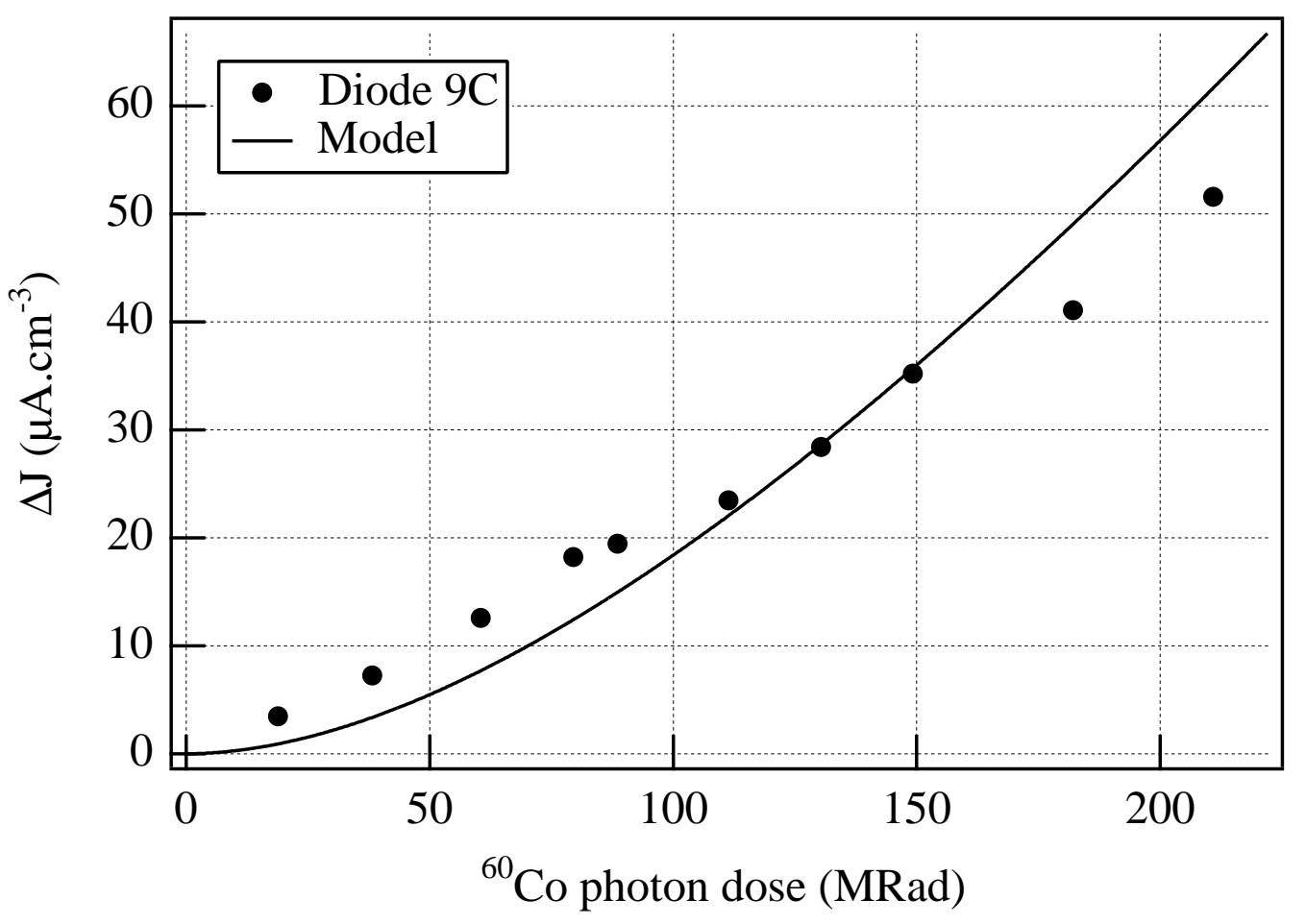

Figure $3 b$ 


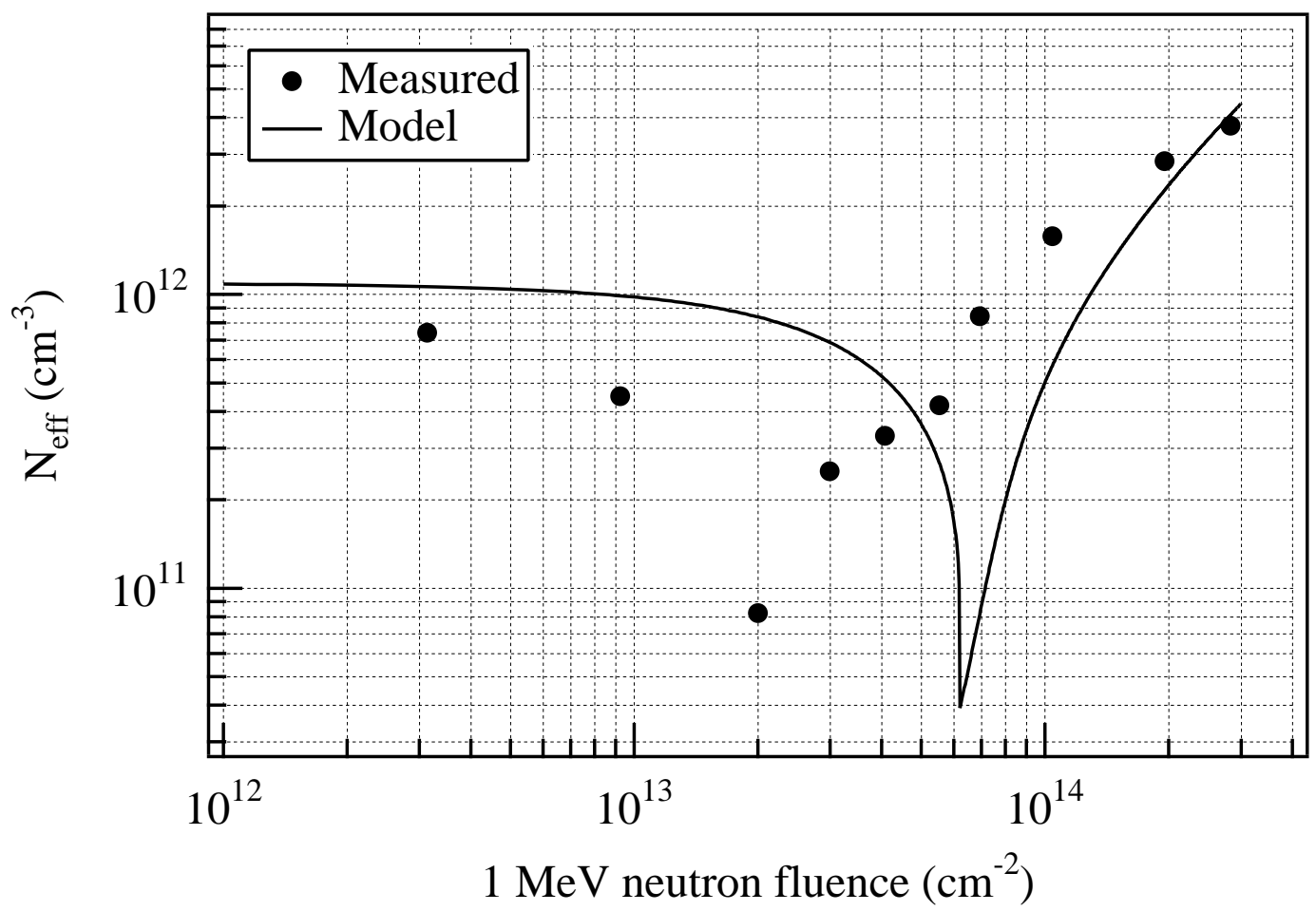

Figure 4a 


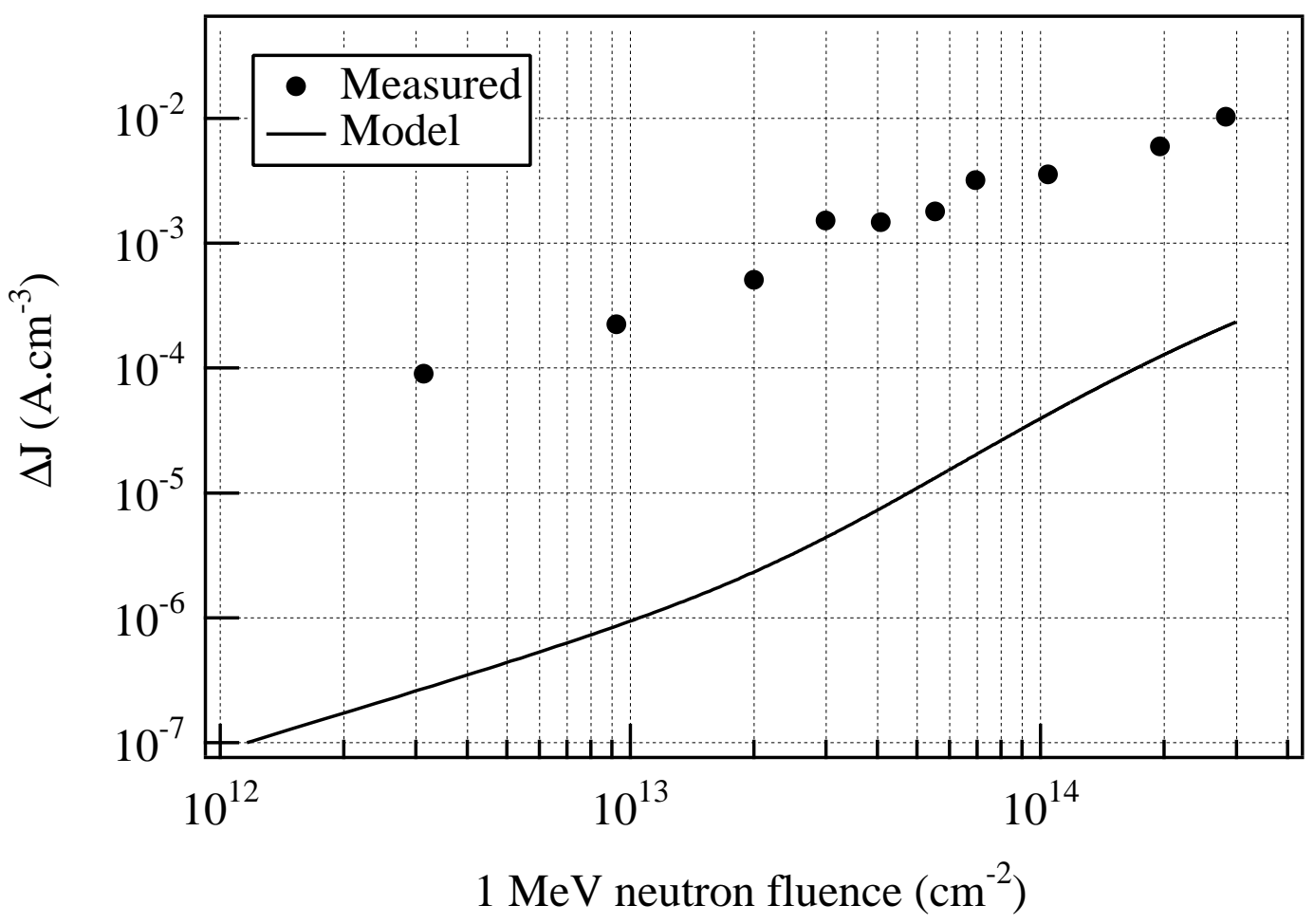

Figure $4 b$ 


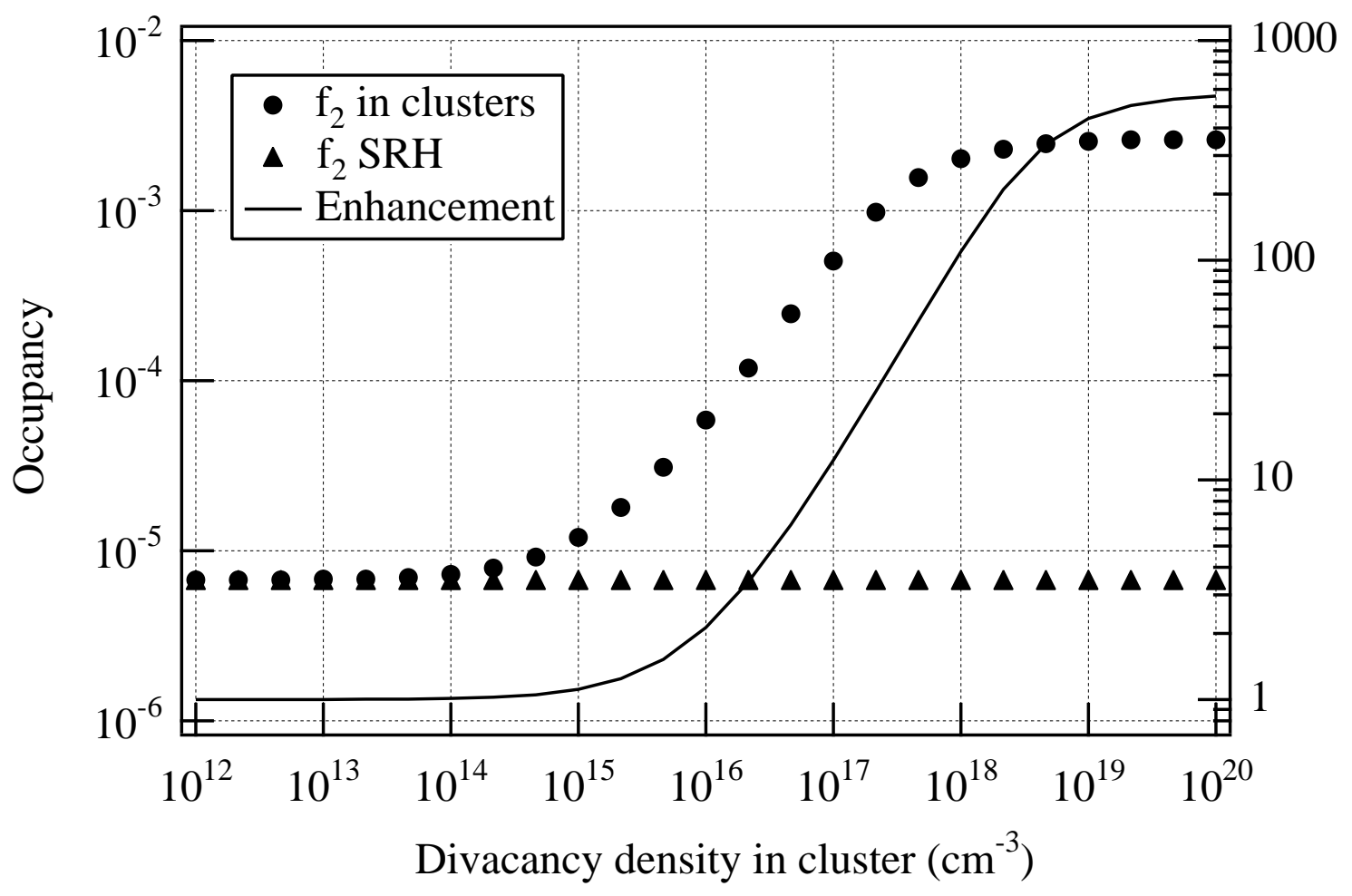

范

Figure 5a 


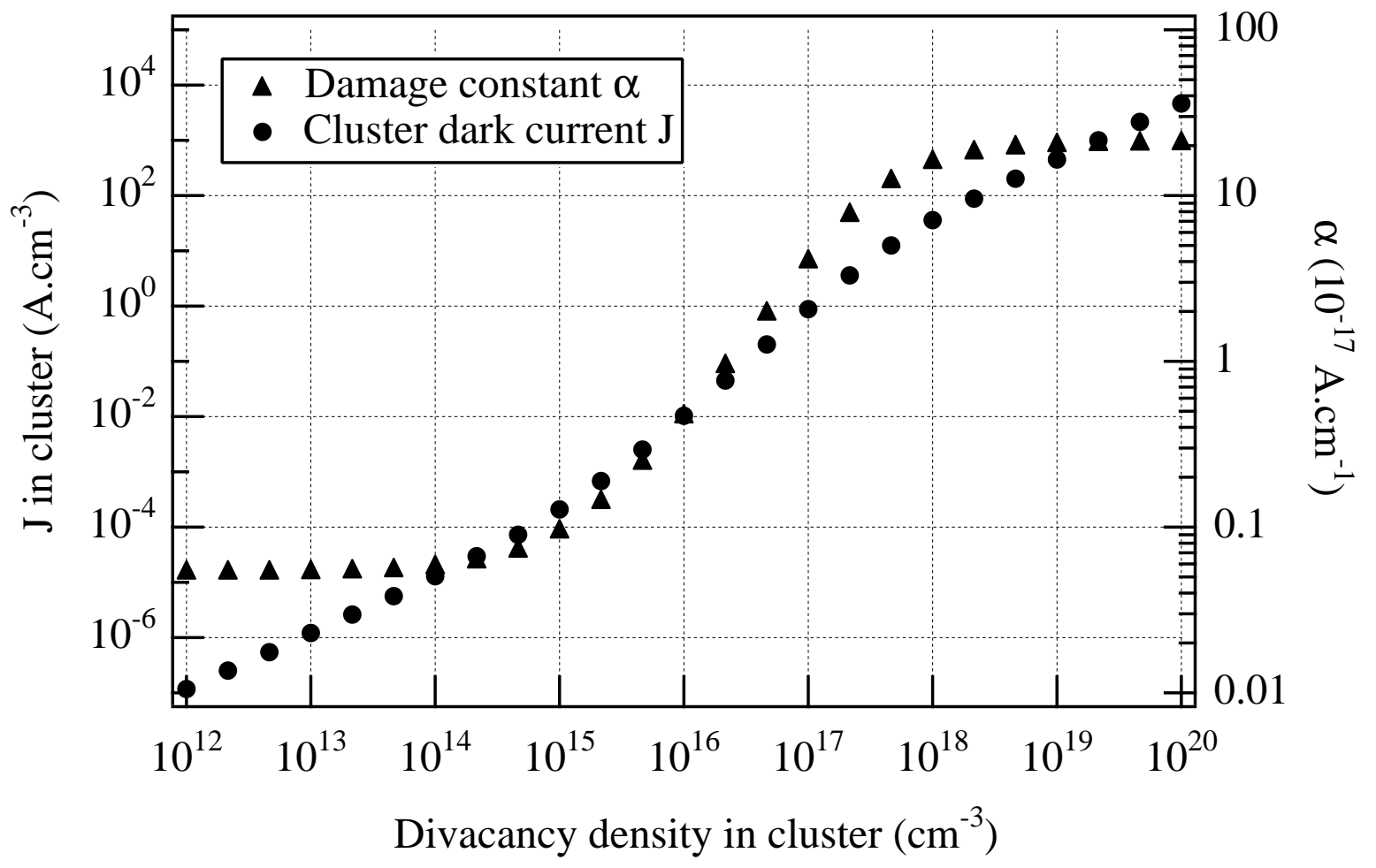

Figure $5 b$ 